\title{
ANÁLISE QUALITATIVA DA ALIMENTAC̣ÃO E O COEFICIENTE INTESTINAL DE METYNNIS CF. ROOSEVELTI EIGENMANN (CHARACIDAE, MYLEINAE), DA LAGOA REDONDA, NÍZIA FLORESTA, RIO GRANDE DO NORTE, BRASIL
}

\author{
Hélio de Castro Bezerra Gurgel ${ }^{1}$ \\ Raimunda Gonçalves de Almeida ${ }^{1}$ \\ Geraldo Barbieri ${ }^{2}$
}

\begin{abstract}
QUANTITATIVE ANALYSIS OF FEEDING AND THE INTESTINAL COEFFICIENT in Metynnis Cf. Roosevelt Elgenmann (Characidae, Myleinae), from Lagoa Redonda, Nizia Floresta, Rio Grande do Norte. Brazil. This species showed feeding activity all year long. Although zooplancton, protozooplaneton and other organisms have been found in the stomaches, this species can be considered, broadly speaking, herbivorous since a great amount of seaweed was found in its diet. Fulness of the stomachs was not associated with sex difference. Full stomachs were found throughout the period. Variations according to the estimated periods were not found. KEY WORDS. Metynnis, intestinal coefficient, feeding activity
\end{abstract}

É conhecida e bastante ressaltada a deficiência alimentar, sobretudo protéica, da população do Nordeste, que se constitui num dos grandes problemas dos governos municipais, estaduais e federal. As contínuas e prolongadas estiagens acarretam redução da oferta dos produtos alimentares e elevação dos preços pecuários impedindo as populações de baixa renda de suprir satisfatoriamente suas necessidades proteicas. Em razão disso, é imperiosa a exploração racional dos recursos hídricos disponíveis na região, como meio de garantir a subsistência dessa faixa da população.

Face a essas circustâncias, várias providências são inseridas nos planos de ação governamental, tais como a melhor utilização do espaço físico, com aumento da produção e produtividade do setor primário, visando amenizar o problema. Neste aspecto, o pescado que representa uma fonte proteica muito valiosa, certamente contribuirá para o suprimento das deficiências alimentares e melhoria de vida dessa população, não somente nos dias de hoje, mas também do futuro.

O presente trabalho tem por objetivo fornecer informações sobre a alimentação de Metynnis cf. roosevelti da Lagoa Redonda, Nízia Floresta, Rio Grande do Norte.

1) Departamento de Botânica, Ecologia e Zoologia, Centro de Biociências, Universidade Federal do Rio Grande do Norte, Campus Universitário, 59072-970 Natal, Rio Grande do Norte, Brasil.

2) Departamento de Hidrobiologia, Universidade Federal de São Carlos, Caixa Postal 676, 13565-905 São Carlos, São Paulo, Brasil. 


\section{MATERIAL E MÉTODOS}

No período de abril de 1987 a março de 1988 foram realizadas coletas quinzenais utilizando-se anzóis de diversos tamanhos, peneiras, redes de espera de 10 e 20 metros de comprimento, com malhas de $1,0,1,5$ e 2,5 cm entre nós e tarrafas de vários tamanhos de malha. As redes de espera permaneceram instaladas durante 24 horas. Dos 2132 exemplares coletados foram registrados os seguintes caracteres: a) Comprimento total (Lt) em centímetros - distância entre a extremidade anterior da maxila e a extremidade do lobo superior da nadadeira caudal, ligeiramente distendida; b) Comprimento do intestino (Li) - medido desde a válvula pilórica até a extremidade do reto - em centímetros; c) Peso total (Wt) e peso do Estômago (We) em gramas.

A fím de se obter uma visão geral da alimentação foi utlizado o método de ocorrência, onde são registrados todos os ítens componentes da dieta, independente do seu número. O total de alimentos encontrados no estômago, foi considerado como tendo um volume de $100 \%$ e em seguida foram estimados os valores percentuais dos diversos ítens alimentares.

A identificação detalhada dos gêneros de algas foi realizada pelas professoras Marisa Roque da Fonseca da Universidade Federal do Rio Grande do Norte e Lúcia Helena Sipaúba da Universidade Estadual Paulista.

Para o conhecimento de alguns aspectos da alimentação procurou-se efetuar antes das análises do conteúdo estomacal, uma avaliação dos graus de repleção, como meio de verificar as possíveis variações na atividade alimentar da espécie. $\mathrm{Na}$ análise sazonal da dieta alimentar adotou-se dois critérios: estômagos sem alimento e estômagos com alimento.

A variação do hábito alimentar foi analisada em função do período estacional chuvoso (março a agosto) e seco (setembro a fevereiro).

A semelhança trófica entre os períodos, chuvoso e seco, foi avaliada pela aplicação do quociente de similaridade proposto por SORENSEN (1948), como segue: $Q S=\frac{2 j .100}{a+b}$ sendo $-\mathrm{QS}=$ quociente de similaridade de SORENSEN; $\mathrm{a}=$ número de espécies no habitat $\mathrm{A}$, ou número de levantamentos com a espécie $\mathrm{a} ; \mathrm{b}=$ número de espécies no habitat $\mathrm{B}$, ou número de levantamentos com a espécie $\mathrm{b} ; \mathrm{j}=$ número de espécies encontradas em ambos os habitats ou o número de levantamentos contendo, simultâneamente, as duas espécies.

\section{RESULTADOS}

Do total de 2132 exemplares examinados, $1869(87,66 \%)$ apresentaram estômagos com alimento e 263 (12,34\%) apresentaram-no sem alimento (Fig. 1A).

Analisando-se a ocorrência de estômagos com e sem alimento, por período chuvoso e seco, e por sexo grupado observa-se que estes são muito próximos: $11,90 \%$ e $12,68 \%$, respectivamente (Figs 1B, 1C).

Por não terem sido verificadas variações qualitativas no espectro alimentar 
de fêmeas e machos, durante o período de estudo, as análises foram consideradas conjuntamente.

(A)

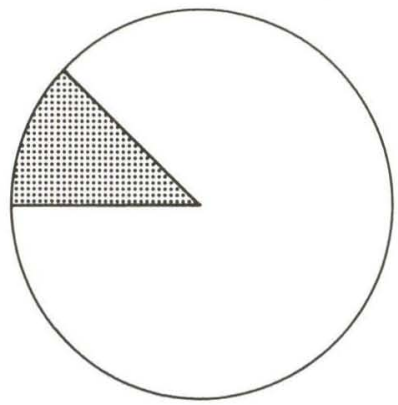

SEM ALIMENTO

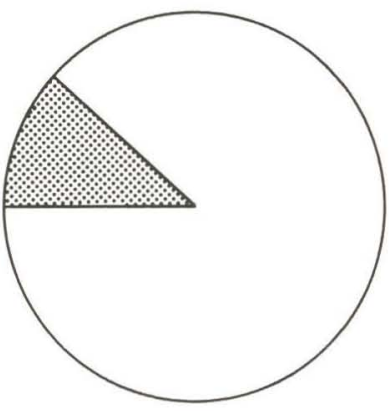

(B)

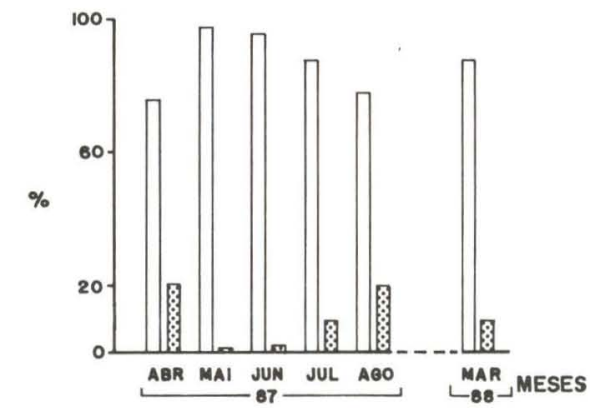

(C)

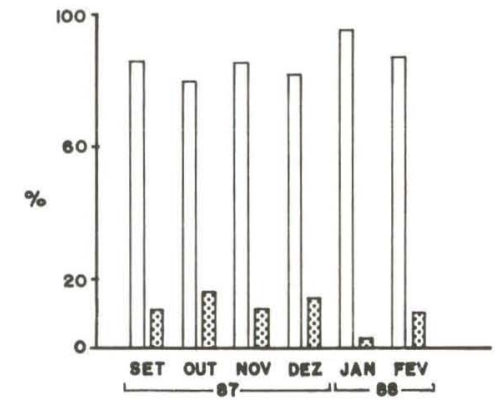

Fig. 1. Distribuição de frequências relativas de estômagos de Metynnis cf. roosevelti, com e sem alimento, por sexo grupado, para o total do período (A) e para os períodos chuvoso (B) e seco (C).

Mensalmente, em termos qualitativos, a dieta apresentou pequenas variações e, desta forma, a análise foi feita de maneira mais sumarizada a fim de que tais resultados pudessem ser claramente visualizados. Para tanto, objetivando 
verificar a existência ou não de influência sazonal na composição da alimentação, determinou-se a ocorrência dos itens alimentares por período chuvoso e seco, uma vez que, além de uma atividade estritamente fisiológica, a dieta também responde às manifestações do meio amhiente (Figs 2A, 2B).
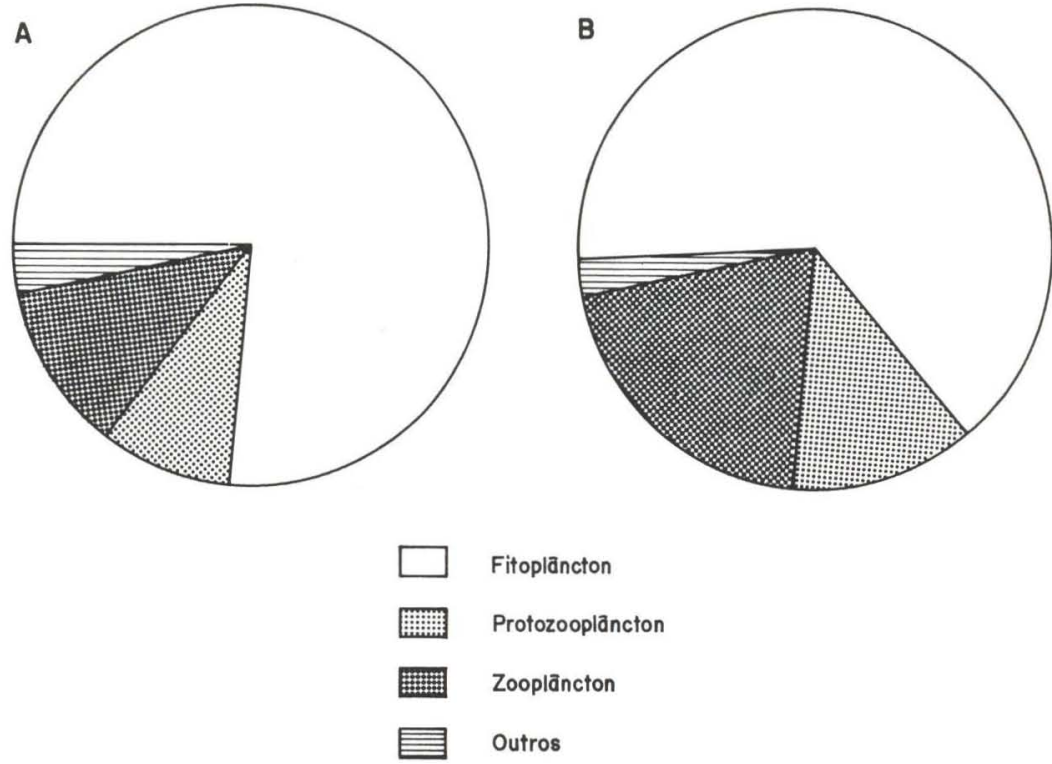

Fig. 2. Distribuição de freqüência relativa de oxorrência de cada item alimentar na composição da dieta de Mtynnis cf. roosevelti, durante os períodos chuvoso (A) e seco (B).

Os itens alimentares identificados até a categoria taxonômica possível, calculados conforme SORENSEN (1948), estão relacionados na figura 3. O fitoplâncton, representado pelas algas foi o item mais expressivo e manteve-se presente durante todo o período (chuvoso e seco), representando $68,0 \%$ do total dos itens. Dentre as algas, os componentes da Divisão Chlorophyta ocorrem com $58,0 \% \mathrm{em}$ relação às demais classes desta categoria.

Com relação ao Zooplâncton, ocorrência em torno de $17,0 \%$ do total dos itens, os Cladoceras destacam-se sobre os Copepoda, Conchostraca. Ostracoda e Rotifera, fracamente representados nos dois períodos.

O Protozooplâncton representado pelas amebas tecadas, compreende uma ocorrência em torno de $12,0 \%$ do total do itens observados.

Na categoria "Outros", que representou o grupo trófico de menor expressividade $(3,0 \%)$ foram reunidos, de maneira geral, fragmentos isolados de macrófitas, sedimentos, exúvias e larvas de insetos. Os sedimentos encontrados podem ter sido oriundo do conteúdo das presas ingeridas.

A figura 4 expressa que o valor do quociente intestinal médio estimado para as classes de comprimento total dos exemplares é de 4,656. 

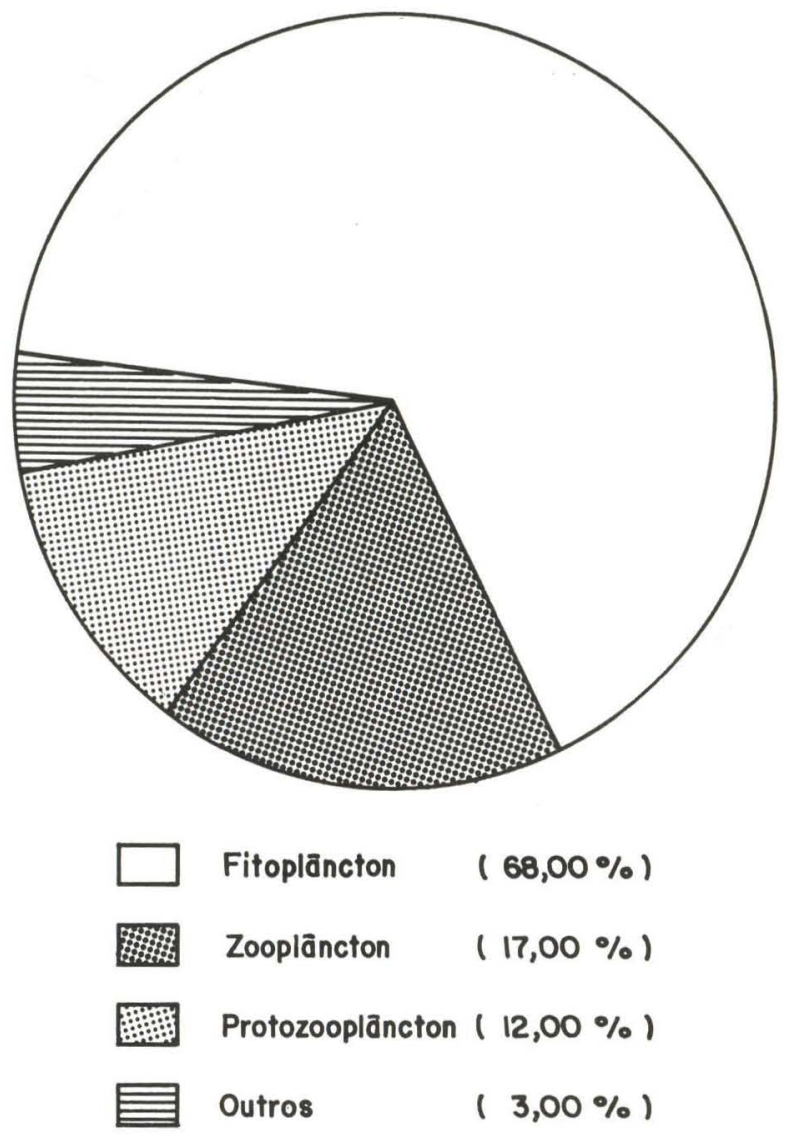

Fig. 3. Distribuição de frequência relativa de ocorrência de cada item alimentar na composição da dieta de Metynnis cf. roosevelti, durante o período de estudo.

\section{DISCUSSÃO E CONCLUSÕES}

Nas regiões tropicais, a maior parte dos trabalhos sobre alimentação de peixes discutem as suas alterações em função das variações estacionais, expressos por períodos alternados de inverno e verão, por serem estes fatores reguladores da disponibilidade, distribuição e comportamento da grande maioria dos organismos.

No Nordeste do Brasil, a grande maioria dos ecossistemas aquáticos têm na distribuição irregular das precipitações pluviométricas, seu ponto fundamental. Assim, enquanto no período chuvoso, ocorre uma ampliação e até mesmo ressurgimento dos corpos d'água, com conseqüente incremento e aumento das fontes alimentares disponíveis, favorecendo a atividade alimentar de diversas espécies; na seca, ocorre o inverso. 


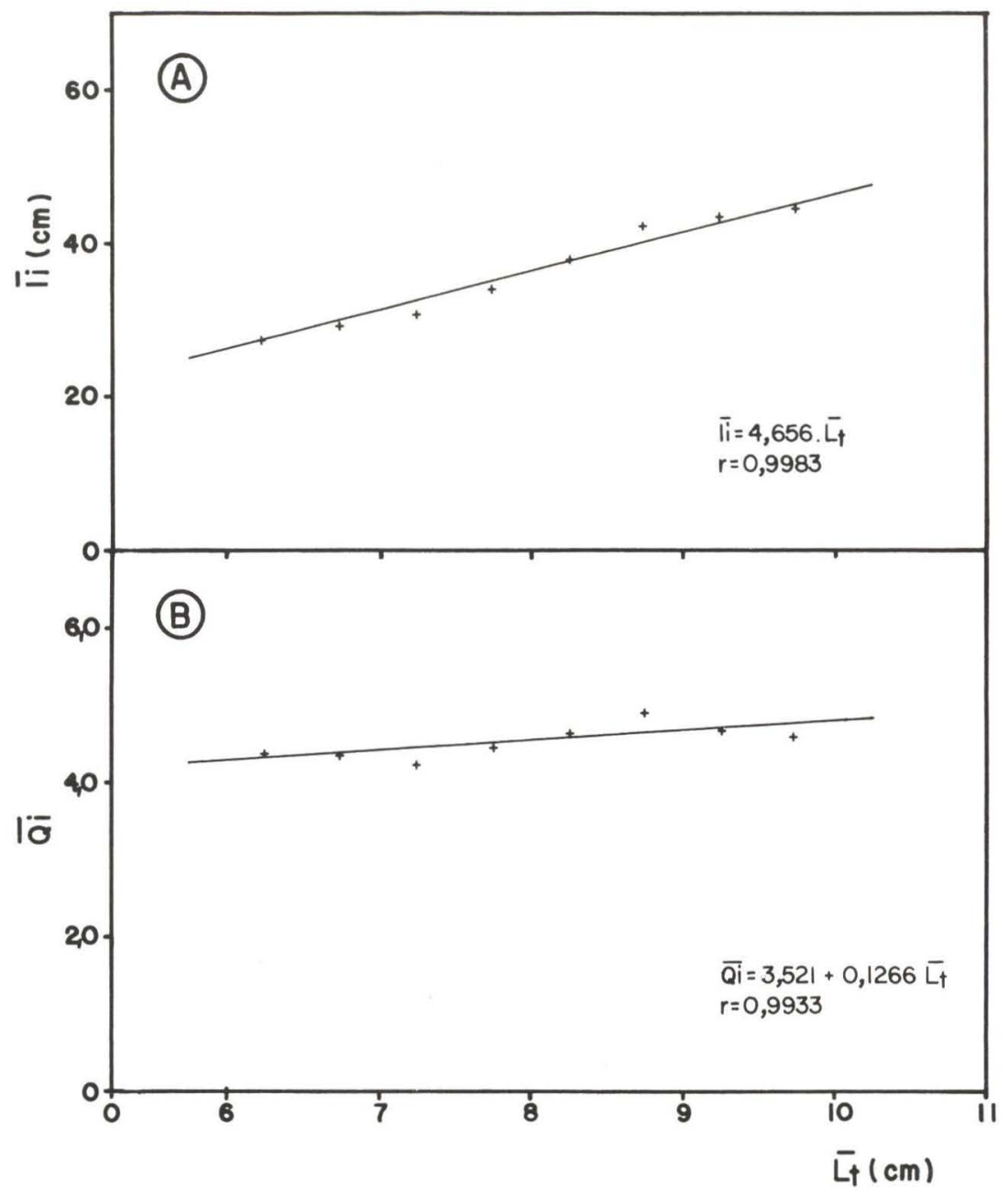

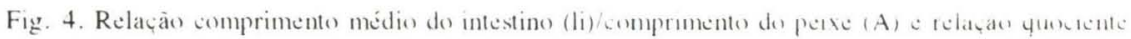
intestinal médio (Qi)/comprimento de exemplares adultos de Metymis ef. roosevelti.

HAHN (1991), acredita que a quantidade de alimento no estômaģo dos peixes pode estar afetado por outros fatores como condições ambientais, metodologia de captura e estado fisiológico do indivíduo.

A análise da atividade alimentar por intermédio do grau de enchimento dos estomagos (com conteúdo e sem conteúdo) foi realizada por período chuvoso e seco. Em relação ao grau de enchimento do trato digestivo em peixes BERG (1979), relata ser este uma boa indicação das condições dentro do nicho, refletindo o nível de competição por alimento, a disponihilidade de organismos alimento, a saúde dos peixes e os fatores ahióticos vigentes. 
Os resultados aqui analisados, evidenciam alterações pouco pronunciadas na atividade alimentar, quando considerados os períodos chuvoso e seco.

MilleR (1967) considera difícil a interpretação da influência da temperatura sobre a atividade alimentar dos peixes, uma vez que as reações das várias espécies à ação daquele fator variam consideravelmente. WHITFIELD \& BLABER (1979), ponderam que, em regiões tropicais e sub-tropicais, as temperaturas de inverno são relativamente altas, permitindo uma alimentação contínua durante todas as estações.

Diana (1979) infere que muitas populações se alimentam continuamente ou no mínimo, tem um período diário de atividade alimentar prolongado. MATALLANAS (1982), estudando o hábito alimentar de Scymnorhinus licha, constatou que esta espécie apresentou altas taxas de estômagos com alimento durante todo o ano. Resultados semelhantes foram obtidos por PAYNE (1975) estudando um ciprinídeo Barbus liberiensis, da região tropical (África).

Os resultados aqui apresentados corroboram os dos autores citados, mostrando que a espécie ora em estudo alimenta-se continuamente ao longo do período amostrado e sugerem que as variações na tomada de alimentos não são afetadas pela temperatura e sazonalidade (período chuvoso e seco), estando, provavelmente relacionados à disponibilidade de alimento. Deve-se no entanto considerar o período reprodutivo.

Analisando-se os resultados deste estudo quanto à composição da dieta de Metynnis cf. roosevelti, observou-se que fitoplâncton foi o item mais consumido. A presença de organismos planctônicos no conteúdo estomacal da espécie, pode se constituir num indicador de que a mesma procura migrar verticalmente para a superfície à procura de alimento, embora possa explorar com relativo sucesso as regiões bentônicas do litoral do lago, explicada pelo consumo de outros organismos, como larvas de diptera, conchostroca e ostracoda, associados à vegetação marginal. Estes resultados corroboram com àqueles obtidos por LOWE-MCCONNELL (1975) em lagos amazônicos, quando relata que no conteúdo estomacal de um mesmo peixe, pode ser encontrado tanto organismos de superfície, quanto de fundo, sugerindo assim que, mesmo espécies adaptadas a uma determinada zona, podem explorar as diversas camadas da massa líquida na tomada de alimentos.

A rigor, poder-se-ia considerar Metynnis cf. rooseveltii como planctófaga, em virtude de ter consumido fitoplâncton e zooplâncton. Contudo, levando-se em consideração a preferência da espécie por Clorophyta e Cyanophyta, pode-se considerá-la como fitófaga, o que concorda com a classificação alimentar atribuída, ainda que de forma generalizada, por GÉRY (1977) aos representantes da subfamília Myleinae, os quais são considerados como vegetarianos.

A maioria dos gêneros de algas encontradas no conteúdo estomacal da espécie em questão ocorre durante todo o período, independentemente, de variações estacionais. Assim, os resultados obtidos, demonstram apenas pequenas variações em relação à disponibilidade de certos gêneros de algas, destacando-se entre outros as Clorophyta pela variedade de táxons.

Sabe-se que uma das adaptações do organismo ao regime alimentar se 
exerce sobre a estrutura e comprimento do conduto gastrintestinal (ANGELESCU \& GNERI 1949; BREEDER \& CRAWFORD 1922 in PAIVA 1959).

HYKES \& MORAVEK (1933) comentam que o regime vegetariano induz o alongamento do tubo digestivo, o carnívoro o encurtamento, e o omnívoro os tamanhos intermediários.

O coeficiente intestinal médio estimado para adultos da espécie em estudos é considerado alto. De acordo com os resultados obtidos pode-se inferir que os exemplares da espécie possuem hábito herbívoro.

De acordo com NIKOLSKY (1963) os peixes que apresentam intestino longo são herbívoros e/ou iliófagos; nos herbívoros o comprimento do intestino é mais de $100 \%$ superior ao comprimento do corpo do peixe. Estas semelhanças foram observadas em Curimatus elegans, Plecostomus paulinis, Plecostomus strigaticeps, Plecostomus hermanni, Plecostomus regani. Plecostomus fluviatilis, Plecostomus nigromaculatus (NomUra et. al. 1981).

\section{REFERÊNCIAS BIBLIOGRÁFICAS}

Angelescu, V., \& F.S. GNerI. 1949. Adaptaciones del aparato digestivo al regimen alimentício en algunos peces del rio Unuguay y del rio de La Plata. Revista del Instituto Nacional de Investigacion de las Ciencias Naturales 1 (6): 161-281.

BERG, J. 1979. Discussion of methods of investigating the food of fishes with reference to a preliminary study of the prey of Gobiusculus flavescens (Gobiidae). Marine Biology 50: 263-273.

Breeder, C.M. \& D.R. CRAWford. 1922. The food of certain minnows. A study of seasonal distary cycle of six Cyprinoids with special reference to fish culture. Zoological, N. Y., 2: 287-327.

DiANA, J. S. 1979. The feeding pattern and daily ration of a carnivore, the norther pike (Esox lucius). Can. J. Zool. 57: 2121-2127.

Eigenmann, C. 1915. The Serrasalminae and Mylinae. Ann. Carneg. Mus., Pittshurg. 9: 226-272.

GÉRY, J. 1977. Characoids of the world. U.S.A. T.H.F., Publications Inc. L.td. , $672 \mathrm{p}$.

Hahn, N.S. 1991. Alimentação e dinâmica da nutrição da curvina Plagioscion squamosissimus (Heckel, 1840) (Pisces, Perciformes) e aspectos da estrutura trófica da ictiofauna acompanhante no rio Paraná. Tese de Doutorado, não publicada, Universidade Estadual Paulista, Rio Claro, 287p.

HYKES, O.V. \& F.R. MORAVEK. 1933. Influence du régime alimentaire sur la longueur du tube digestif des poiss ons. Compt. Rend. Seanc. Soc. Biologie, Paris, 113: 1239-1241.

LOWE-MCCONNEL, R.H. 1975. Fish communities in tropical freshwater: their distribution, ecology and evolution. London, Longman, 337p.

Matallanas, J. 1982. Estudio del régime alimentario de Lampanyctus crocodilus (Méditerrâneo Occidental). Tethys 10 (3): 254-260. 
MilleR, P.J. 1961. Age, growth and reproduction of the rocky gody, Gobius paganellus L., in the Isle of Man. J. Mar. Biol. Ass. U.K. 41: 737-769.

NikOLSKY, G.V. 1963. The ecology of fishes. London, Academic Press, 352p.

Nomura, H.; L. Nemoto \& I.M.M. Mueller. 1981. Alimentação de seis espécies de peixes do gênero Plecostomus walbaum 1782 (Pisces, Loricariidae), do rio Mogi Guaçu, SP. In: Sem. Reg. Eco., n. 2, Resumos, São Carlos, p. 389-405.

PaIVA, M.P. 1959. Notas sobre o crescimento, do tubo digestivo e a alimentação da Gitubarana, Salminus hilarii Val., 1829 (Pisces, Characidae). Bol. Mus. Nac., n.s. Zoo., Rio de Janeiro, 196: 1-23.

PAYNE, A.I. 1975. The reprodutive cycle, condition and feeding in Barbus liberiensis, a tropical stream-dwelling cyprinid. J. Zool. Lond. 176: 247-269.

SORENSEN, T. 1948. A method of stablishing graps of equal amplitude in plant sociology basead an similarity of species. Biol. Skr. 5 (1): 34.

WhITFIELD, A.K., BLABER, S.J.M. 1978. Food and feeding ecology of piscivourous fishes at lake St Lucia, Zulaland. J. Fish. Biol. 13: 675-691.

Recebido em 15.I.1994; aceito em 14.VII.1994. 